\title{
The research on OCT deep cavity three-dimensional morphology of application of endoscopic
}

\author{
Hongchang Ding, Guohua Cao
}

Changchun University of Science and Technology

\begin{abstract}
Keywords: OCT (optical coherence tomography), industrial endoscope, the three-dimensional topography detection, the galvanometer.
\end{abstract}

\begin{abstract}
In order to explore the three-dimensional topography information of complex deep cavity wall, making use of the imaging principle based on optical coherence tomography (OCT). Building up the experiment system by combining the OCT with endoscope. Developing the related system platform. It is verified that testing on the three-dimensional appearance characteristics of deep cavity wall by combining the OCT with endoscope through operation of the experimental system if feasible.
\end{abstract}

\section{Introduction}

It causes the defects in the tiny pipe deep cavity walls such as maturing, pitting, crack, peeling, perforation, function holes blocked and so on due to the reasons of medium corrosion and mechanical shock. It needs to detect the in-wall pipeline in order to ensure the safety of small pipe being used with fully functional. In current detection methods, it is difficult to meet the testing requirements even not impossible to achieve when there is water mist or reservoir on tiny pipe wall. This paper puts forward a new kind of detection scheme which solving the problems by combining the OCT system with industrial endoscope, and verifying the feasibility of this method.

\section{Theory of OCT}

OCT first found a niche in imaging the retina, since no other imaging modality could provide non-contact, cross-sectional images of the retina with high resolution. These images enabled physicians to detect and monitor macular diseases including age-related macular degeneration and glaucoma, and OCT is now a standard of care in ophthalmology.

OCT (Optical coherence tomography) is a form of Optical signal acquisition and processing. The system has simple structure, low developmentcosts, no damage, the intervention, high resolution, and high sensitivity and fast scanning, which makes the advantages of the optical scattering medium such as biological tissues can be scanned. The $3 \mathrm{~d}$ image resolution can reach micron grade.

The OCT system includes a low coherent light source, interference system, sample arm, the reference arm and detector and other devices. A low coherent light from the light source is divided into two parts when goes through the optical fiber coupler, which goes into the reference arm and sample arm. Reflected light from the reference arm and sample arm meets again on the fiber coupler. It occurs interference and detects the light intensity, then respectively into the sample and the reference arm. When two arms returns the optical path difference of light is in the light, the beam of light in the optical fiber coupling interference occurs, form interference of light contains information can be measured, which can be photoelectric detected. It can get needed information by processing the probe on the resulting interference signals.

The mathematics of optical interferometry can be simply demonstrated using the example of light traveling as a plane wave, described by equation 1 , where the wave is traveling in the $\hat{z}$ direction and the electric field is pointed in the $\hat{x}$ direction. (The magnetic field, which has the same form, is $90^{\circ}$ out of phase with the electric field and points in the $-\hat{y}$ direction.)

$\hat{x} E=\hat{x} E_{\circ} \cos (k z-\omega t+\emptyset)(1)$

In equation $1, E_{\circ}$ is the electric field amplitude in Volts per unit length, $\mathrm{k}$ is the circular wavenumber or $\frac{2 \pi}{\lambda}$ where $\lambda$ is the wavelength of light, and $\omega$ is the angular frequency ( $\omega=2 \pi v$ where $v$ 
is the frequency of oscillation). The circular wavenumber $k$ and angular frequency $\omega$ are related to the speed of light $V$ by $\mathrm{V}=\frac{\omega}{\mathrm{k}}$. In a vacuum, $V$ is constant for all wavelengths of light, $\mathrm{V}_{\text {vacuum }}=c=$ $3 \times 10^{8} \mathrm{~m} / \mathrm{s}$. The speed of light is approximately constant in air, and most applications, includingOCT, assume $\mathrm{V}_{\text {air }}=\mathrm{V}_{\text {vacuum }}=c$.



Fig 1 Simple theory of OCTFig 2 the principle diagram of the system

OCT technology can obtain two-dimensional images with high resolution, which three dimensional data sets can be established by a continuous adjacent two-dimensional image. Getting the three-dimensional morphology characteristics of the measured sample through $3 \mathrm{~d}$ reconstruction algorithm.

The light interference of two partially coherent beams in OCT system can be expressed by the change of the light intensity.

$$
\mathrm{I}=k_{1} I_{s}+k_{2} I_{s}+2 \sqrt{\left(k_{1} I_{s}\right) \cdot\left(k_{2} I_{s}\right)} \cdot R_{e}[\gamma(\tau)](2)
$$

In the formula, $\mathrm{I}_{\mathrm{s}}$ is on behalf of the light intensity, $\mathrm{k}_{1}+\mathrm{k}_{2}<1$ is on behalf of coherent light segregation ratio, $\gamma(\tau)$ is on behalf of degree of coherence. This function is a complex number, which is dependent on the proportion of the envelope and carrier by the interference of the light scanning from the reference arm (time delay tau)

$$
\gamma(\tau)=\exp \left[-\left(\frac{\pi \Delta v \tau}{2 \sqrt{\ln 2}}\right)^{2}\right] \cdot \exp \left(-j 2 \pi v_{0} \tau\right)
$$

In the formula, $\Delta v$ is on behalf of the spectrum width of light source, $v_{0}$ is on behalf of the center of the light source frequency. In the equation (3), Gaussian function envelope is the scope of the light carrier wave modulated. The peak values of the envelope is the position of the microstructure in the samples have been tested. The amplitude is dependent on the reflection of the sample surface. The frequency of the optical carrier generates Doppler Effect by the influence of the interferometer arms move when it is scanning. The frequency can be controlled by the scanning speed. In this way, the movement of the interferometer arms has two effects. By changing the length of light path to achieve depth scanning and optical carrier with Doppler frequency shift. In optical coherence tomography, Doppler frequency shift can be expressed as the following.

$$
f_{\text {Dopp }}=\frac{2 \cdot v_{0} \cdot v_{s}}{c}(4)
$$

In the formula, $\mathrm{v}_{0}$ is on behalf of the center of the light source frequency. $\mathrm{v}_{\mathrm{S}}$ is on behalf of the light path changing scanning speed, $c$ is on behalf of velocity of light.

It is independent of the resolution between the axial and lateral of optical interference in optical coherence tomography.

$$
\mathrm{l}_{\mathrm{c}}=\frac{2 \ln 2}{\pi} \cdot \frac{\lambda_{0}{ }^{2}}{\Delta \lambda} \approx 0.44 \cdot \frac{\lambda_{0}{ }^{2}}{\Delta \lambda}(5)
$$

III. The principle of system

As shown in the schematic of this system, a laser light emitted by low coherence illuminant through the 2x2 fiber coupler into use as a reference laser and laser samples, of which $90 \%$ of the light given to measuring arm for sample detection and $10 \%$ as a reference laser. Reference arm primarily as a precision scanning of the reference mirror for generating a reference light, the reference light is 
directly reflected into the reference arm mirror, the reference arm moves laterally in practical applications, the movement range is $\pm 5 \mathrm{~mm}$.

This detection system component in the sample is detected at the sample arm by scanning galvanometer used in conjunction with the endoscope. Scanning galvanometer placed on the back end of the endoscope, the sample light incident fiber coupler to the system's scanning galvanometer that scanning galvanometer produce swing then swinging through the endoscope, the sample light is focused onto the sample through the endoscope for testing on the detection of sample.

Sample light incident on the surface of the sample, the light reflected by the sample and the reference arm mirror reference light reflected back through the optical fiber coupler to a CCD camera at the confluence. Since the light source is weak coherence, the reflected light only from a specific depth of the sample could coherent reference light, and the reflected light from the sample at different depths with different phase delay, as the delay increases, the interference signal is a sharp decline. A position corresponding to the reference arm, the reference arm only when the mirror is reflected back the reflected reference light detected sample signal light match the optical path difference in order to produce interference with the reference arm optical path length difference between coherent signals optical interference will not occur with the reference light. Lateral movement of the reference arm; adjust the position of the reference arm mirror, so we can obtain a tomographic image of the sample depth. Under the sample arm of the two-dimensional scanning galvanometer undergoing a series of high-speed scanning, the information of the signal under test sample obtained after being treated properly.

When light is scanned over the sample, the spectrometer splitting system will expand into an interference pattern by the CCD detector spectral information to convert it into an electric signal. Passing through the interface computer to realize acquisition of spectral data. After computer processing spectral data extracted from the sample under test standard description of the information. Together with OCT rotary scanning probe can get a two-dimensional image of the sample. Computer record the reference outline and sample morphology during exercise testing one by one. The interference signal is detected by the photo detectors, the optical interference by the detector into an electric signal after the data acquisition and processing system through the collection, and analyzed on computer.

\section{Experiment}

Through the theory of surface is stained with oil samples for testing to verify the rationality of the system. Before the experiment will be stained with greasy dirt on the surface of the measured paste have multilayer cellophane tape bring further confirms the feasibility of the experiment.

This system is made up of OCT rotated scanning probe, endoscopy, scanning galvanometer, low coherence light source, CCD camera, optical couplers and other components. Apply with $850 \pm 30 \mathrm{~nm}$ tunable light source quality fiber laser, Thorlabs's company's GVS002 dimensional scanning galvanometer system can achieve full mechanical scan range of $\pm 12.5^{\circ}$ angular resolution of $0.0008^{\circ}$ (15 micro radians), the second into two $90 / 10$ fiber optic splitter, linear CCD camera and spectrograph as a major test of the original.



Fig 3 Experimental apparatus figure 
The result of this test can be clearly expressed by the PC software, as shown below, the information collected by the CCD pass through to the computer by spectrometer. Computer analysis and processing can be clearly found that the number of white stripes to fall in the upper part of the picture for the OCT within surface morphology of the DUT endoscope combined information systems detected

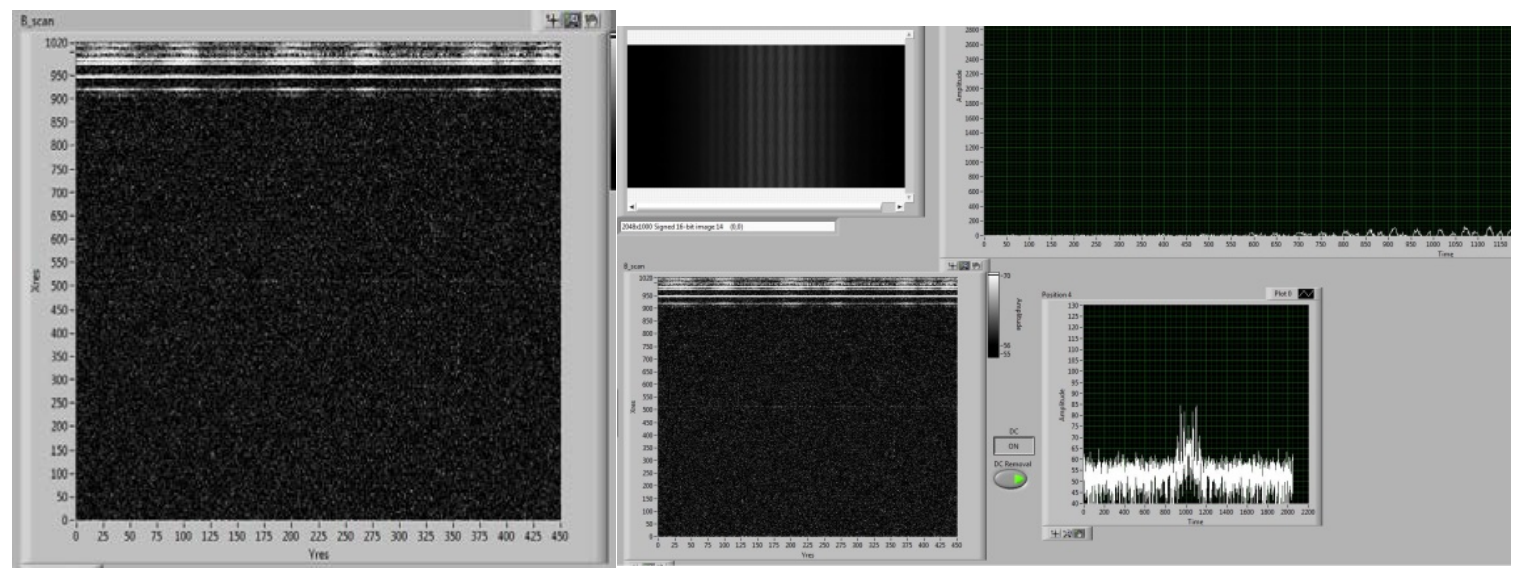

Fig 4 the experimental test resultsFig 5 Operations display

In the figure 5 operations display position 4 the process of experiment. It describes the time domain images.

\section{Conclusion}

This article describes the technical of OCT combined with endoscopic. Propose the OCT with endoscope combined with the principle of the diagram. Completed the deep cavity wall surface detection system construction and succeed in the presence of oil and water droplets case sample surface topography acquisition. As can be seen, morphology combined with endoscopic OCT technology through ordinary means of detection cannot be achieved, such as the presence of oil and other characteristics of the DUT deep cavity wall surface to detect and provide the basis for future studies of the deep.

\section{References}

[1]Huang D, Swanson E A,Lin C P, et al ..,“Optical coherence tomog raphy.Science, 1991, 254 (22) ":1178-1181.

[2]J. Rogowska,N.A. Patel, J. G. Fujimoto, et al., "Optical coherencetomographic elastography technique for measuring deformation and strain of atherosclerotic tissues," Heart 90, 556 (2004)

[3]J.M. Zara, S. Yazdanfar, K.R. Rao, J.A. Izatt, S.W. Smith, "Electrostatic micromachine scanning mirror for optical coherence tomography”, Opt. Lett. 28 (8), 628 (2003)

[4]MEADWAY A,DARBRAZI S H H,DOBRE G,et al.“A rapid method of measuring dispersion in low coherence interferometry and optical coherence tomography systems,"Journal of Optics,2010,12(1):015302.

[5]Shi G H,Rao X J,Ding Zh H,et al..,"Distance measurement of optical surface based on optical cpherence tomography,”Chinese Journal of Laser,2009,36(2):210-214.(in Chinese)

[6]Tuqiang Xie,Huikai Xie,G.K.Fedder,et al, Endoscopic optical coherence tomography with new MEMS mirror, Electronics Letters,2003,39(21).

[7]U. Morgner, W. Drexler, F_ X. KMner, et aL "Spectroscopic optical coherence tomography," Opt. Lett.,2000, 25: 111-113 